\title{
Differentiating Crohn's disease from intestinal tuberculosis at presentation in patients with tissue granulomas
}

\author{
G Watermeyer, MB ChB, FCP, Cert Gastroenterology (SA), MPH; S Thomson, ChM, FRCS (Ed \& Eng), FRCP (Ed) \\ Division of Gastroenterology, Department of Medicine, Groote Schuur Hospital and Faculty of Health Sciences, University of Cape Town, \\ South Africa
}

Corresponding author: G Watermeyer (gillian.watermeyer@uct.ac.za)

Background. Overlapping clinical, endoscopic, radiographic and histological features, coupled with poor microbiological yield, make differentiating Crohn's disease (CD) from intestinal tuberculosis (ITB) challenging. Granulomas are present in both diseases; in CD they predict the need for immunosuppressive therapy that requires ITB to be excluded before initiation.

Objectives. To compare granuloma-positive CD and ITB, to identify factors that may aid in diagnosis.

Methods. This was a retrospective cohort study evaluating granuloma-positive CD and ITB identified from a pathology database.

Results. Sixty-eight ITB and 48 CD cases were identified. Patients with ITB were more likely to be male, and to have HIV infection, isolated colitis, night sweats and tachycardia. ITB was also associated with lower serum albumin and haemoglobin and higher C-reactive protein levels, a chest radiograph showing active tuberculosis, and lymph nodes $>1 \mathrm{~cm}$ on imaging. Extraintestinal manifestations (EIMs) were predictive of $\mathrm{CD}$. There were no significant differences in smoking status, symptom duration or perianal disease. On multivariate analysis, HIV positivity (odds ratio (OR) 29.72, 95\% confidence interval (CI) $2.15-410.96$; $p=0.01$ ), isolated colitis (OR 6.17, $95 \%$ CI 1.17 - 32.52; $p=0.03$ ) and the absence of EIMs (OR $0.10,95 \%$ CI $0.01-0.65 ; p=0.02$ ) remained significant risk factors for ITB.

Conclusion. This is the first study to identify clinical and biochemical factors to aid in differentiating granuloma-positive ITB from CD. EIMs support a diagnosis of CD, while isolated colitis and HIV are predictors of ITB.

S Afr Med J 2018;108(5):399-402. DOI:10.7196/SAMJ.2018.v108i5.13108

Crohn's disease $(\mathrm{CD})$ is a chronic inflammatory disorder that can affect any part of the gastrointestinal tract (GIT), notably the terminal ileum and caecum. The major differential diagnosis of $\mathrm{CD}$ in our setting in Cape Town, South Africa (SA), is intestinal tuberculosis (ITB). Both disorders share overlapping clinical, endoscopic, radiographic and histological features, notably the presence of tissue granulomas. Coupled with a poor microbiological yield in ITB, this makes differentiating these diseases challenging. Traditional diagnostic modalities such as acid-fast bacilli (AFB), Mycobacterium tuberculosis (MTB) culture, tuberculin skin testing, interferongamma release assays and chest radiographs are often negative in ITB ${ }^{[1]}$ In addition, polymerase chain reaction (PCR) testing of the GIT for MTB is not validated in our setting and is not currently recommended or approved. Given the very high incidence and prevalence of MTB infection in SA, a diagnosis of CD is only made once ITB has been excluded. This often requires an empirical trial of anti-tuberculosis (anti-TB) therapy. ${ }^{[1,2]}$

Misdiagnosis leads to delays in initiating effective therapy for CD, while the use of potent immunomodulators (IMMs) and biologicals in the setting of ITB can have fatal consequences. It is therefore important to make an accurate diagnosis at the earliest possible stage.

The differentiation of ITB from CD is particularly problematic in patients with non-caseating granulomas, as these suggest a very real possibility of ITB. ${ }^{[3]}$ To date, no study has been done in this particular subgroup to analyse factors that could aid in this diagnostic dilemma.

\section{Objective}

To compare patients with granuloma-positive CD and ITB, to identify variables that could aid in differentiating between the two conditions.

\section{Methods}

The research protocol was approved by the Ethics Committee of the University of Cape Town (ref. no.833/2014). A retrospective cohort study (2005 - 2015) was conducted evaluating adult patients with granuloma-positive CD or ITB. Subjects were identified from a pathology database and information was extracted from patient folders and laboratory and radiology records.

The following data were collected at presentation: demographics, clinical, biochemical, histological and radiographic variables, and HIV status (Tables 1 - 4).

The diagnosis of CD was made on the basis of a combination of clinical, radiological, endoscopic and histological features as per the European Crohn's and Colitis Organisation consensus statement. $^{[4]}$

ITB was diagnosed if any of the following were present: AFB positivity on tissue biopsy, positive culture for MTB, and full response after completion of anti-TB treatment.

\section{Statistical analysis}

The association between all baseline risk factors and the diagnosis of $\mathrm{CD}$ and ITB was assessed by univariate analysis. Normally distributed continuous variables are expressed as means (standard deviations (SDs)). Continuous variables that were not normally distributed are expressed as medians and interquartile ranges (IQRs). Categorical variables were compared using the $\chi^{2}$ test, or Fisher's exact test when appropriate. Variables with $p$-values $<0.05$ were further tested in a series of logistic multivariate regression models. The most parsimonious model was selected. The analysis was performed using Stata version 11 (StataCorp, USA). 


\section{Results}

Sixty-eight patients with ITB and 48 with CD with granulomas were identified. Of those with ITB, 42 (61.8\%) had AFB present on Ziehl-Neelsen staining and 36 (52.9\%) were culture-positive for MTB. At presentation (Table 1), patients with ITB were younger than those with $\mathrm{CD}(p=0.03)$, and more likely to be male $(p=0.03)$ and of black ethnicity $(p<0.0001)$. Isolated colonic involvement was significantly more common in ITB than in CD $(p=0.04)$. There were no significant differences in smoking status, symptom duration or perianal disease.

Clinical variables associated with a diagnosis of ITB (Table 2) were a history of night sweats $(p=0.01)$ and tachycardia $(p<0.0001)$ at presentation. Patients with $\mathrm{CD}$ were significantly more likely to have extraintestinal manifestations (EIMs) than those with ITB $(p<0.0001)$. The commonest EIM in CD was iron deficiency anaemia (IDA). The only other two EIMs of note were erythema nodosum ( $n=4$ cases) and uveitis $(n=2)$, all in patients with CD. Other symptoms such as diarrhoea, abdominal pain and weight loss were not significant. Similarly, fever, the presence of an abdominal mass on palpation and ascites on clinical examination were not predictive.

Thirty-three percent of patients with ITB had a chest radiograph with features of active tuberculosis (TB); this was predictive of concurrent ITB $(p<0.0001)$. In contrast, radiographic features suggesting past $\mathrm{TB}$ were not. Overall, only $62.1 \%$ of the cohort had undergone cross-sectional imaging at presentation (Table 4). Of the radiographic abnormalities reported, only lymph nodes $>1 \mathrm{~cm}$ in size and ascites were predictive of ITB. There were no significant differences with regard to the presence of central node attenuation, splenic abscesses, inflammatory masses or intraabdominal collections.

Thirty-five patients with ITB (51.5\%) were HIV-positive, as opposed to a single patient in the CD group. HIV positivity was significantly associated with a diagnosis of ITB $(p<0.0001)$. The mean (SD) CD4+ count in the HIV-positive patients was 269 (250) cells/ $\mu \mathrm{L}$.

ITB was also associated with lower serum albumin $(p<0.0001)$ and haemoglobin $(p<0.0001)$ values at presentation than $\mathrm{CD}$, as well

Table 1. Patient demographics at presentation

\begin{tabular}{|c|c|c|c|}
\hline & $\mathrm{CD}(N=48)$ & ITB $(N=68)$ & $p$-value \\
\hline Duration of symptoms before diagnosis (months), median (IQR) & $9.5(4-12)$ & $3(1-8)$ & 0.06 \\
\hline Age (years), median (IQR) & $28(19-24)$ & $35(26-44)$ & 0.03 \\
\hline Gender (female), $n(\%)$ & $32(66.7)$ & $31(45.6)$ & 0.03 \\
\hline Self-declared ethnicity, $n(\%)$ & & & $<0.0001$ \\
\hline White & $7(14.5)$ & $3(4.4)$ & \\
\hline Coloured & $36(75.0)$ & $29(42.7)$ & \\
\hline Black & $5(10.4)$ & $35(51.5)$ & \\
\hline Asian & $0(0.0)$ & $1(1.5)$ & \\
\hline Current smoker, $n(\%)$ & $23(47.9)$ & $24(35.3)$ & 0.11 \\
\hline Site of involvement, $n(\%)$ & & & 0.04 \\
\hline Ileum & $27 / 45(60.0)$ & $28 / 67(41.8)$ & \\
\hline Colon & $5 / 45(11.1)$ & $17 / 67(25.4)$ & \\
\hline Ileum and colon & $10 / 45(22.2)$ & $10 / 67(14.9)$ & \\
\hline Upper GIT & $3 / 45(6.7)$ & $5 / 67(7.5)$ & \\
\hline Isolated perianal disease & $0 / 45(0.0)$ & $7 / 67(10.5)$ & \\
\hline Any perianal disease & $11 / 45(24.4)$ & $12 / 67(17.9)$ & 0.40 \\
\hline Previous TB, $n(\%)$ & $2 / 45(4.4)$ & $11 / 68(16.2)$ & 0.11 \\
\hline
\end{tabular}

\section{Table 2. Clinical features at presentation}

\begin{tabular}{|c|c|c|c|}
\hline & $\mathrm{CD}(N=48)$ & ITB $(N=68)$ & $p$-value \\
\hline Night sweats, $n(\%)$ & $4 / 45(8.9)$ & $21 / 67(31.3)$ & 0.01 \\
\hline Abdominal cramps, $n(\%)$ & $39 / 45(86.7)$ & $49 / 67(73.1)$ & 0.09 \\
\hline Nausea and vomiting, $n(\%)$ & $26 / 45(57.8)$ & $34 / 60(56.7)$ & 0.58 \\
\hline Bowel action, $n(\%)$ & & & 0.92 \\
\hline Normal & $18 / 45(40.0)$ & $26 / 67(38.8)$ & \\
\hline Diarrhoea & $20 / 45(44.4)$ & $32 / 67(47.8)$ & \\
\hline Constipation & $7 / 45(15.6)$ & $9 / 67(13.4)$ & \\
\hline EIMs, $n(\%)$ & $17 / 45(37.8)$ & $3 / 67(4.5)$ & $<0.0001$ \\
\hline Loss of weight, $n(\%)$ & $31 / 45(68.9$ & $51 / 67(76.1)$ & 0.4 \\
\hline Weight loss (kg), median (IQR) & $7.5(5-10)$ & $10(5-15)$ & 0.54 \\
\hline Temperature $\left({ }^{\circ} \mathrm{C}\right)$, median (IQR) & $36(36-37)$ & $36.8(35.6-37.9)$ & 0.70 \\
\hline Pulse rate (bpm), median (IQR) & $80(76-90)$ & $100(80-115)$ & 0.001 \\
\hline Pulse rate $>100 \mathrm{bpm}, n(\%)$ & $5 / 44(11.4)$ & $35 / 66(53.0)$ & $<0.0001$ \\
\hline Palpable abdominal mass, $n(\%)$ & $6 / 45(13.3)$ & $9 / 67(13.4)$ & 0.10 \\
\hline Ascites on examination, $n(\%)$ & $0 / 45(0.0))$ & $2 / 64(3.1)$ & 0.23 \\
\hline
\end{tabular}


Table 3. Laboratory parameters at presentation

\begin{tabular}{|c|c|c|c|}
\hline & $\mathrm{CD}(N=48)$ & ITB $(N=68)$ & $p$-value \\
\hline Haemoglobin (g/dL), median (IQR) & $11.5(10.4-12.5)$ & $9.7(8.3-11.8)$ & 0.01 \\
\hline Haemoglobin $>10$ g/dL, $n(\%)$ & $37 / 43(86.1)$ & $29 / 64(45.3)$ & $<0.0001$ \\
\hline Serum albumin $(\mathrm{g} / \mathrm{L})$, median (IQR) & $39(35-42)$ & $30.5(23-36)$ & 0.91 \\
\hline Serum albumin $>30 \mathrm{~g} / \mathrm{L}, n(\%)$ & $37 / 42(88.1)$ & $22 / 42(52.4)$ & $<0.0001$ \\
\hline Platelet count $\left(\times 10^{9} / \mathrm{L}\right)$, median $(\mathrm{IQR})$ & $412(322-533)$ & $411(289-507)$ & 0.43 \\
\hline CRP (mg/L), median (IQR) & $26(10-65)$ & $75(30-109)$ & 0.04 \\
\hline
\end{tabular}

\section{Table 4. Radiographic imaging at presentation}

\begin{tabular}{|c|c|c|c|}
\hline & $\mathrm{CD}(N=48)$ & ITB $(N=68)$ & $p$-value \\
\hline Features of previous TB on CXR, $n(\%)$ & $2 / 45(4.4)$ & $12 / 68(17.7)$ & 0.11 \\
\hline Features of active TB on CXR, $n(\%)$ & $0 / 45(0)$ & $22 / 68(32.4)$ & $<0.0001$ \\
\hline Patients who had cross-sectional imaging, $n(\%)$ & $26 / 45(57.8)$ & $46 / 66(69.7)$ & 0.24 \\
\hline Type of imaging, $n(\%)$ & & & 0.08 \\
\hline Ultrasound & $6 / 26(23.1)$ & $17 / 46(36.9)$ & \\
\hline MRE & $4 / 26(15.4)$ & $1 / 46(2.2)$ & \\
\hline $\mathrm{CT}$ & $16 / 26(61.5)$ & $28 / 46(60.9)$ & \\
\hline Central areas of low attenuation, $n(\%)$ & $0 / 23(0)$ & $5 / 34(14.7)$ & 0.16 \\
\hline An inflammatory mass, $n(\%)$ & $16 / 26(61.5)$ & $26 / 46(56.5)$ & 0.54 \\
\hline Ascites, $n(\%)$ & $0 / 26(0)$ & $9 / 46(29.6)$ & 0.02 \\
\hline Splenic abscesses, $n(\%)$ & $0 / 26(0)$ & $2 / 34(5.9)$ & 0.23 \\
\hline Lymph nodes $>1 \mathrm{~cm}, n(\%)$ & $1 / 26(3.9)$ & $16 / 46(34.8)$ & 0.004 \\
\hline Intra-abdominal collections, $n(\%)$ & $5 / 26(19.2)$ & $8 / 46(17.4)$ & 0.79 \\
\hline
\end{tabular}

as higher C-reactive protein (CRP) levels ( $p=0.04$ ) (Table 3). Ninety percent of cases of anaemia in ITB were secondary to anaemia of chronic disease. On multivariate analysis, HIV positivity (odds ratio (OR) 29.72, 95\% confidence interval (CI) 2.15 - 410.96; $p=0.01$ ), isolated colonic disease (OR 6.17, 95\% CI $1.17-32.52 ; p=0.03$ ) and the absence of EIMs (OR 0.10, 95\% CI $0.01-0.65$; $p=0.02$ ) remained significant risk factors for ITB. The presence of these three risk factors yielded $93 \%$ specificity for the diagnosis of ITB. On receiver operating characteristic curve analysis, the area under the curve for a model of these three risk factors was 0.88 (Fig. 1).

\section{Discussion}

Differentiating ITB from CD is notoriously challenging, as they share numerous clinical, endoscopic and radiographic features. Both diseases can affect the GIT at any point from the mouth to the anus, with a predilection for the ileocaecal region. Both may present with perianal disease, intestinal strictures or fistulas, and in addition they share many EIMs. Distinguishing ITB from CD in SA is further complicated by very high rates of MTB infection. Although most cases are pulmonary, extrapulmonary TB can occur, and the GIT is commonly affected. ${ }^{[5,6]}$

Treating undiagnosed ITB with potent immunosuppressive CD medications can have potentially fatal consequences, particularly the risk of dissemination. If the diagnosis is unclear, common practice is therefore to give a trial of anti-TB therapy and assess the clinical response. ${ }^{[2]}$ This approach is far from ideal, as it delays implementation of appropriate $\mathrm{CD}$ medication, which may affect long-term outcomes. $\mathrm{CD}$ is a progressive and destructive illness that evolves over time to be complicated by the development of strictures, fistulas or abscesses. Ultimately the majority of patients require surgery. ${ }^{[7]}$ Aggressive medical therapy with IMMs such as

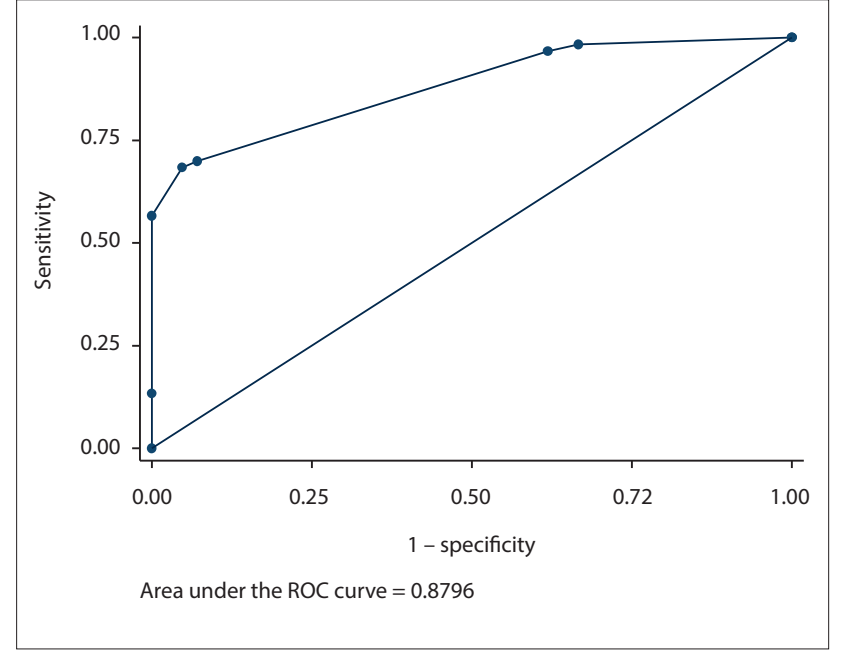

Fig. 1. ROC curve analysis for the model including EIMs, HIV and isolated colonic disease. The presence of these three risk factors yielded $93 \%$ specificity for the diagnosis of ITB. On ROC analysis, the AUC for a model of these three risk factors was 0.88. (ROC = receiver operating curve; EIMs = extraintestinal manifestations; ITB = intestinal tuberculosis; $A U C=$ area under the curve.)

azathioprine or methotrexate and biologicals may alter this natural history and improve long-term outcomes. ${ }^{[6]}$ However, to be effective these should be introduced early in the disease course, ideally at diagnosis, before the development of irreversible complications. ${ }^{[6]}$

Several studies have analysed factors early in the course of $\mathrm{CD}$ that may predict future outcomes and identify patients at risk of developing complicated $\mathrm{CD}$ who would receive greatest benefit 
from early, aggressive therapy. ${ }^{[6]}$ One such predictor is the presence of tissue granulomas on histological examination. A recent cohort study in the Western Cape Province, SA, showed a two-fold increase in the risk of developing complicated $\mathrm{CD}$ in those with this finding at diagnosis. ${ }^{[8]}$

The differentiation of ITB from CD is even more challenging in patients with histological evidence of non-caseating granulomas, as this further heightens the suspicion of ITB. ${ }^{[3]}$ Patients with ITB are significantly more likely to have non-caseating granulomas than those with $\mathrm{CD}^{[1,3]}$ This was highlighted in a recent meta-analysis of 38 studies differentiating CD from ITB. There were 2117 cases of $\mathrm{CD}$ and 1589 cases of ITB. The authors used the data to construct a Bayesian model to predict the probability of ITB v. CD. In this analysis, the presence of granulomas was significantly associated with a diagnosis of ITB. ${ }^{[3]}$

Ours is the first study with the objective of identifying factors differentiating ITB from CD in exclusively granuloma-positive patients. Several factors were identified as being valuable in this regard.

On multivariate analysis, the presence of EIMs at diagnosis strongly suggested a diagnosis of $\mathrm{CD}$. This is in concordance with reports from several cohort studies, as well as the formerly mentioned Bayesian model. ${ }^{[3,9]}$

The commonest EIM in CD was IDA. This finding is not unexpected, as it is the most common systemic complication of CD. ${ }^{[10]}$ The other reported EIMs in the CD patients were erythema nodosum and uveitis.

Several studies have shown that the presence of perianal disease strongly supports a diagnosis of CD. Interestingly, this was not seen in our study. In fact, in our cohort all seven cases of isolated perianal disease were proven to be TB. It is possible that the prevalence of perianal TB is underestimated. A 2009 prospective study from our institution evaluated 96 patients with perianal fistulas. TB was present in $7.3 \%$ of cases, suggesting that this is not an uncommon manifestation of TB in our setting. ${ }^{[1]}$

In our study, significantly more patients with ITB than CD had isolated colonic involvement (25.4\% v. $11.1 \%)$. This is higher than in other series, where only $10 \%$ of patients demonstrated this finding. ${ }^{[12]}$ However, the incidence of colonic involvement was increased in immunocompromised patients and in those with HIV. ${ }^{[12]}$ In our cohort, $50.1 \%$ of patients with isolated colonic involvement were HIV-infected. This could explain the high incidence of this location.

Other variables supporting a diagnosis of ITB, such as anaemia, hypoalbuminaemia, tachycardia and higher median CRP levels, were no longer significant on multivariate analysis. This probably reflects their association with systemic toxicity and the inflammatory burden seen in HIV-TB co-infection, as they were no longer significant after adjusting for HIV status.

Not unexpectedly HIV, infection was shown to be the strongest risk factor for ITB. It is well recognised that the burden of TB in SA is fuelled by the HIV epidemic, with 7 million South Africans infected with the virus. ${ }^{[13]} \mathrm{SA}$ is currently home to $22.5 \%$ of all individuals with TB and HIV co-infection across the globe. ${ }^{[13]}$

Interestingly, in our study patients with granulomatous ITB-HIV co-infection had a mean CD4+ count of 269 cells/ $\mu \mathrm{L}$, suggesting that granuloma-positive ITB, which is difficult to differentiate from $\mathrm{CD}$, requires a degree of immune competence. This is supported by data showing that reductions in CD4+ counts in co-infected persons were associated with both poorer granuloma formation and higher bacterial load. ${ }^{[14]}$

\section{Study limitations}

Given the retrospective nature of this study, there were some missing data points. In addition, there were some disease characteristics that were poorly documented, on which availability of more detail would have enhanced the analysis. These were the number and morphology of granulomas, tuberculin skin testing, the endoscopic mucosal appearance, the number of diseased colonic segments, and features on routine cross-sectional imaging. These would have been useful additional tools, as they have been shown to have good diagnostic accuracy in differentiating CD from ITB. ${ }^{[15]}$

\section{Conclusion}

Excluding ITB is essential when considering potent immunosuppressive therapies for $\mathrm{CD}$. This study focused on subjects with tissue granulomas at diagnosis, as these predict a severe course of $\mathrm{CD}$. Several clinical and biochemical factors were identified that will aid in making the correct diagnosis, notably HIV infection, which was the strongest risk factor for ITB in this study. Isolated colonic involvement was also associated with ITB, while EIMs predicted a diagnosis of $\mathrm{CD}$. To our knowledge, this is the first study to restrict analysis to granuloma-positive patients, the subgroup in which differentiating CD from ITB is most challenging. These data will aid in ensuring a timely diagnosis and reduce the risk of delayed $\mathrm{CD}$ treatment, as well as misdiagnosing ITB.

\section{Acknowledgements. None.}

Author contributions. GW was responsible for study design, data collection, statistical analysis, and preparation of the manuscript. ST critiqued and modified the final document.

Funding. None.

Conflicts of interest. None.

1. Epstein D, Watermeyer G, Kirsch R. Review article: The diagnosis and management of Crohn's disease in populations with high-risk rates for tuberculosis. Aliment Pharmacol Ther 2007;25(12):1373-1388. https://doi.org/10.1111/j.1365-2036.2007.03332.x

2. Ooi CI, Hilmi I, Makharia GK, et al. The Asia Pacific Consensus Statements on Crohn's Disease. Part 1: definition, diagnosis and epidemiology. J Gastroenterol Hepatol 2015;31(1):45-55. https://doi. 1: definition, diagnosis and epidemiology. J Gastroenterol Hepatol 2015;31(1):45-55. https://doi.
org/10.1111/jgh.12956

3. Limsrivilai J, Shreiner AB, Pongpaibul A, et al. Meta-analytic Bayesian model for differentiating intestinal tuberculosis from Crohn's disease. Am J Gastroenterol 2017;112(3):415-427. https://doi. org/10.1038/ajg.2016.529

4. Gomollón F, Dignass A, Annes V, et al. 3rd European Evidence-based Consensus on the Diagnosis and Management of Crohn's Disease 2016: Part 1: Diagnosis and medical management. J Crohns Colitis 2017;11(1):3-25. https://doi.org/10.1093/ecco-jcc/jjwl68

5. Donoghue HD, Holton J. Intestinal tuberculosis. Curr Opin Infect Dis 2009;22(5):490-496. https://doi. org/10.1097/QCO.0b013e3283306712

6. Torres J, Mehandru S, Colombel JF, et al. Crohn's disease. Lancet 2017;389(10080):1741-1755. https:// doi.org/10.1016/S0140-6736(16)31711-1

7. Beaugerie L, Seksik P, Nion-Larmurier I, et al. Predictors of Crohn's disease. Gastroenterology 2006;130(3):650-656. https://doi.org/10.1053/j.gastro.2005.12.019

8. Watermeyer G, Thomson SR. Granulomas at initial diagnosis of Crohn's disease signals a poor outcome. S Afr Med J 2015;105(6):480-483. https://doi.org/10.7196/SAMJ.9093

9. Singh B, Kedia S, Konijeti G, et al. Extraintestinal manifestations of inflammatory bowel disease and intestinal tuberculosis: Frequency and relation with disease phenotype. Indian J Gastroenterol 2015;34(1):43-50. https://doi.org/10.1007/s12664-015-0538-7

10. Filmann N, Rey J, Schneeweiss S, et al. Prevalence of anemia in inflammatory bowel diseases in European countries: A systematic review and individual patient data meta-analysis. Inflamm Bowel Dis 2014;20(5):936-945. https://doi.org/10.1097/01.MIB.0000442728.74340

11. Stupart D, Goldberg P, Levy A, et al. Tuberculous anal fistulas-prevalence and clinical features in an endemic area. S Afr J Surg 2009;47(4):116-118.

12. Debi U, Ravisankar V, Prasad KK, et al. Abdominal tuberculosis of the gastrointestinal tract: Revisited. World J Gastroenterol 2014;20(40):14831-14840. https://doi.org/10.3748/wjg.v20.i40.14831

13. World Health Organization. Global Tuberculosis Report 2016. Geneva: WHO, 2016. www.who.int/tb/ publications/global_report/gtbr2016_annex2.pdf (accessed 6 January 2018).

14. Diedrich CR, O'Hern J, Wilkinson RJ. HIV-1 and the Mycobacterium tuberculosis granuloma: A systematic review and meta-analysis. Tuberculosis 2016;98:62-76. https://doi.org/10.1016/j.

15. Kedia S, Sharma R, Nagi B, et al. Computerized tomography-based predictive model for differentiation of Crohn's disease from intestinal tuberculosis. Indian J Gastroenterol 2015;34(2):135-143. https://doi. org/10.1007/s12664-015-0550-y

Accepted 12 February 2018. 